\title{
Stability of Stochastic Differential Equations with Distributed and State-Dependent Delays
}

\author{
Leonid Shaikhet \\ Department of Mathematics, Ariel University, Ariel 40700, Israel.
}

How to cite this paper: Leonid Shaikhet. (2020) Stability of Stochastic Differential Equations with Distributed and State-Dependent Delays. Journal of Applied Mathematics and Computation, 4(4), 181-188.

DOI: 10.26855/jamc.2020.12.009

Received: September 22, 2020

Accepted: October 28, 2020

Published: December 3, 2020

*Corresponding author: Leonid Shaikhet, Department of Mathematics, Ariel University, Ariel 40700, Israel.

Email: leonid.shaikhet@usa.net

\begin{abstract}
Stability of a linear stochastic differential equation with distributed and state-dependent delays is investigated. Sufficient conditions of asymptotic mean square stability are obtained via the general method of Lyapunov functionals construction and the method of linear matrix inequalities (LMIs). Numerical simulations illustrate the theoretical results and open a new unsolved problem of the obtained stability conditions improving.
\end{abstract}

\section{Keywords}

Stochastic differential equation, State-dependent delays, Asymptotic mean square stability, Linear matrix inequalities (LMIs), Unsolved problem

\section{Introduction}

Differential equations with delays that are depending on the system state, so called, state-dependent delays, are enough new but at the same time are enough popular in research both in the deterministic case [1, 5-9, 11, 12, 14, 15, 17-20, 22-29, 32-34] and in the stochastic case [2, 3, 21, 30, 36, 37]. However, it is necessary to note that research for stochastic differential equations with state-dependent delays, in particular, with problems of stability for equations of such type, one can meet much less common. In this paper, we try to improve a bit this situation and to show how can be obtained sufficient conditions of asymptotic mean square stability for the linear Ito stochastic differential equation [13] with distributed delays, depending on time and on the system state

$$
\begin{gathered}
d x(t)=\left(A x(t)+\sum_{i=1}^{k} \beta_{i}\left(x_{t}\right)\right) d t+\sum_{i=1}^{m} \gamma_{i}\left(x_{t}\right) d w_{i}(t), \\
\beta_{i}\left(x_{t}\right)=\int_{t-h_{i}(t, x(t))}^{t} B_{i} x(s) d s, \quad \gamma_{i}\left(x_{t}\right)=\int_{t-\tau_{i}(t, x(t))}^{t} C_{i} x(s) d s, \\
x_{0}(s)=\phi(s) \in H_{2}, \quad s \leq 0 .
\end{gathered}
$$

Here, $x(t) \in \mathbf{R}^{n}, \quad A, B_{i}, C_{i} \in \mathbf{R}^{n \times n}, \quad w_{1}(t), \cdots, w_{m}(t)$, are mutually independent standard Wiener processes on a com-

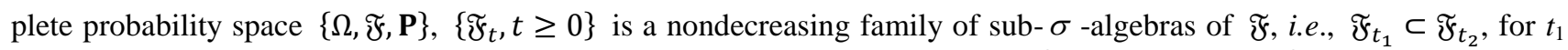
$<t_{2}, H_{2}$ is a space of $\mathfrak{F}_{0}$-adapted stochastic processes $\phi(s) \in \mathbf{R}^{n}, s \leq 0,\|\phi\|^{2}=\sup _{s \leq 0} \mathbf{E}|\phi(s)|^{2}, \mathbf{E}$ is the mathematical expectation with respect to the measure $\mathbf{P}[13,31]$.

Below via the general method of Lyapunov functionals construction [31] sufficient conditions for asymptotic mean square stability of the zero solution of the Eq. (1.1) are obtained in the terms of linear matrix inequalities (LMIs) [4, 10, 35]. 


\subsection{Auailiary definitions and statements}

Definition 1.1 The zero solution of the Eq. (1.1) is called:

- mean square stable if for each $\varepsilon>0$ there exists a $\delta>0$ such that $\mathbf{E}|x(t, \phi)|^{2}<\varepsilon, t \geq 0$, proided that $\|\phi\|^{2}<\delta$; - asymptotically mean square stable if it is mean square stable and $\lim _{t \rightarrow \infty} \mathbf{E}|x(t, \phi)|^{2}=0$ for each initial function $\phi$.

Let $x(t)$ be a value of the solution of the Eq. (1.1) in the time moment $t, x_{t}=x(t+s), s<0$, be the trajectory of the solution of the Eq. (1.1) until the time moment $t$. Consider a functional $V(t, \varphi):[0, \infty) \times H_{2} \rightarrow \mathbf{R}_{+}$that can be presented in the form $V(t, \varphi)=V(t, \varphi(0), \varphi(s)), s<0$, and for $\varphi=x_{t}$ put

$$
V_{\varphi}(t, x)=V(t, \varphi)=V\left(t, x_{t}\right)=V(t, x, x(t+s)), \quad x=\varphi(0)=x(t), \quad s<0 .
$$

Denote by $D$ the set of functionals, for which the function $V_{\varphi}(t, x)$, defined in (1.2), has a continuous derivative with respect to $t$ and two continuous derivatives with respect to $x$. Let ' be the sign of transpose, $\nabla$ and $\nabla^{2}$ be respectively the first and the second derivatives of the function $V_{\varphi}(t, x)$ with respect to $x$. For the functionals from $D$ the generator $L$ of the Eq. (1.1) has the form [13, 31]

$$
L V(t, x)=\frac{\partial V_{\varphi}(t, x(t))}{\partial t}+\nabla V_{\varphi}^{\prime}(t, x(t))\left(A x(t)+\sum_{i=1}^{k} \beta_{i}\left(x_{t}\right)\right)+\frac{1}{2} \sum_{i=1}^{m} \gamma_{i}^{\prime}\left(x_{t}\right) \nabla^{2} V_{\varphi}(t, x(t)) \gamma_{i}\left(x_{t}\right) .
$$

Theorem 1.1 [31] Let there exist a functional $V(t, \varphi) \in D$, positive constants $c_{1}, c_{2}, c_{3}$, such that the following conditions hold:

$$
\mathbf{E} V\left(t, x_{t}\right) \geq c_{1} \mathbf{E}|x(t)|^{2}, \quad \mathbf{E} V(0, \phi) \leq c_{2}\|\phi\|^{2}, \quad \mathbf{E} L V\left(t, x_{t}\right) \leq-c_{3} \mathbf{E}|x(t)|^{2} .
$$

Then the zero solution of the Eq. (1.1) is asymptotically mean square stable.

Lemma 1.1 [31] For arbitrary vectors $a, b \in \mathbf{R}^{n}$ and a positive definite matrix $R \in \mathbf{R}^{n \times n}$ the following inequality holds: $a^{\prime} b+b^{\prime} a \leq a^{\prime} R a+b^{\prime} R^{-1} b$.

Schur complement [16]. The symmetric matrix $\left[\begin{array}{cc}A & B \\ B^{\prime} & C\end{array}\right]$, where $A \in \mathbf{R}^{n \times n}, B \in \mathbf{R}^{n \times m}, C \in \mathbf{R}^{m \times m}$, is negative definite if and only if $C$ and $A-B C^{-1} B^{\prime}$ are both negative definite.

\section{Main result}

We will assume that the delays $h_{i}(t, x)$ and $\tau_{i}(t, x)$ are bounded, i.e., satisfy the conditions:

$$
\begin{aligned}
& 0 \leq h_{i}(t, x) \leq h_{i}, \quad i=1, \cdots, k, \\
& 0 \leq \tau_{i}(t, x) \leq \tau_{i}, \quad i=1, \cdots, m .
\end{aligned}
$$

Note that this assumption includes particular cases of constant delays $\left(h_{i}, \tau_{i}\right)$ and bounded time-varying delays $\left(h_{i}(t), \tau_{i}(t)\right)$.

Theorem 2.1 Let the conditions (2.1) hold and there exist positive definite matrices $P, R_{11}, \cdots, R_{1 k}, R_{21}, \cdots, R_{2 m} \in \mathbf{R}^{n \times n}$ that satisfy the $L M I$

$$
\begin{aligned}
{\left[\begin{array}{cc}
\Phi & \Psi \\
\Psi^{\prime} & R
\end{array}\right]<0, \quad \Phi } & =A^{\prime} P+P A+\sum_{i=1}^{k} h_{i} R_{1 i}+\frac{1}{2} \sum_{i=1}^{m} \tau_{i}^{2} C_{i}^{\prime} R_{2 i} C_{i} \in \mathbf{R}^{n \times n}, \\
\Psi & =\left[\sqrt{h_{1}} B_{1}^{\prime} P \cdots \sqrt{h_{k}} B_{k}^{\prime} P \frac{\tau_{1}}{\sqrt{2}} C_{1}^{\prime} P \cdots \frac{\tau_{m}}{\sqrt{2}} C_{m}^{\prime} P\right] \in \mathbf{R}^{n \times(k+m) n}, \\
R & =\operatorname{diag}\left\{-R_{11}, \cdots,-R_{1 k},-R_{21}, \cdots,-R_{2 m}\right\} \in R^{(k+m) n \times(k+m) n} .
\end{aligned}
$$


Then the zero solution of the Eq. (1.1) is asymptotically mean square stable.

Proof: Following the general method of Lyapunov functionals construction [31], we will construct the Lyapunov functional $V$ in the form $V=V_{1}+V_{2}$, where $V_{1}(x(t))=x^{\prime}(t) P x(t)$ and $V_{2}$ will be chosen below. Via (1.3) we have

$$
\begin{aligned}
L V_{1}(x(t)) & =2 x^{\prime}(t) P\left(A x(t)+\sum_{i=1}^{k} \beta_{i}\left(x_{t}\right)\right)+\sum_{i=1}^{m} \gamma_{i}^{\prime}\left(x_{t}\right) P \gamma_{i}^{\prime}\left(x_{t}\right) \\
& =x^{\prime}(t)\left(A^{\prime} P+P A\right) x(t)+\sum_{i=1}^{k} I_{1 i}+\sum_{i=1}^{m} I_{2 i},
\end{aligned}
$$

where via (1.1)

$$
\begin{aligned}
& I_{1 i}=\int_{\left.t-h_{i}(t, x(t))\right)}^{t}\left(x^{\prime}(t) P B_{i} x(s)+x^{\prime}(s) B_{i}^{\prime} P x(t)\right) d s, \\
& I_{2 i}=\int_{t-\tau_{i(t, x, t(t))}^{t}}^{t} \int_{t-\tau_{i(t, x(t))}}^{t} x^{\prime}(s) C_{i}^{\prime} P C_{i} x(\theta) d \theta d s .
\end{aligned}
$$

Using Lemma 1.1 for $I_{1 i}$ with $R_{1 i}>0, a=x(t), b=P B_{i} x(s)$, via (2.1) we have

$$
\begin{aligned}
I_{1 i} & \leq \int_{t-h_{i}(t, x(t))}^{t}\left(x^{\prime}(t) R_{1 i} x(t)+x^{\prime}(s) B_{i}^{\prime} P R_{1 i}^{-1} P B_{i} x(s)\right) d s \\
& \leq h_{i} x^{\prime}(t) R_{1 i} x(t)+\int_{t-h_{i}}^{t} x^{\prime}(s) B_{i}^{\prime} P R_{1 i}^{-1} P B_{i} x(s) d s .
\end{aligned}
$$

Similarly, using Lemma 1.1 for $I_{2 i}$ with $R_{2 i}>0, a=C_{i} x(s), b=P C_{i} x(0)$, via (2.1) we obtain

$$
\begin{aligned}
I_{2 i} & =\frac{1}{2} \int_{t-\tau_{i}(t, x(t))}^{t} \int_{t-\tau_{i}(t, x(t))}^{t}\left(x^{\prime}(s) C_{i}^{\prime} P C_{i} x(\theta)+x^{\prime}(\theta) C_{i}^{\prime} P C_{i} x(s)\right) d \theta d s \\
& \leq \frac{1}{2} \int_{t-\tau_{i}(t, x(t))}^{t} \int_{t-\tau_{i}(t, x(t))}^{t}\left(x^{\prime}(s) C_{i}^{\prime} R_{2 i} C_{i} x(s)+x^{\prime}(\theta) C_{i}^{\prime} P R_{2 i}^{-1} P C_{i} x(\theta)\right) d \theta d s \\
& \leq \frac{1}{2} \tau_{i} \int_{t-\tau_{i}}^{t} x^{\prime}(s)\left(C_{i}^{\prime} R_{2 i} C_{i}+C_{i}^{\prime} P R_{2 i}^{-1} P C_{i}\right) x(s) d s .
\end{aligned}
$$

From (2.3), (2.4), (2.5) it follows that

$$
\begin{aligned}
L V_{1}(x(t)) \leq & x^{\prime}(t)\left(A^{\prime} P+P A+\sum_{i=1}^{k} h_{i} R_{1 i}\right) x(t)+\sum_{i=1}^{k} \int_{t-h_{i}}^{t} x^{\prime}(s) B_{i}^{\prime} P R_{1 i}^{-1} P B_{i} x(s) d s \\
& +\frac{1}{2} \sum_{i=1}^{m} \tau_{i} \int_{t-\tau_{i}}^{t} x^{\prime}(s) C_{i}^{\prime}\left(R_{2 i}+P R_{2 i}^{-1} P\right) C_{i} x(s) d s .
\end{aligned}
$$

Choosing the additional functional $V_{2}$ in the form

$$
\begin{aligned}
V_{2}\left(x_{t}\right)= & \sum_{i=1}^{k} \int_{t-h_{i}}^{t}\left(s-t+h_{i}\right) x^{\prime}(s) B_{i}^{\prime} P R_{1 i}^{-1} P B_{i} x(s) d s \\
& +\frac{1}{2} \sum_{i=1}^{m} \tau_{i} \int_{t-\tau_{i}}^{t}\left(s-t+\tau_{i}\right) x^{\prime}(s) C_{i}^{\prime}\left(R_{2 i}+P R_{2 i}^{-1} P\right) C_{i} x(s) d s,
\end{aligned}
$$

we obtain

$$
\begin{aligned}
L V_{2}\left(x_{2}\right)= & \sum_{i=1}^{k} h_{i} x^{\prime}(t) B_{i}^{\prime} P R_{1 i}^{-1} P B_{i} x(t)-\sum_{i=1}^{k} \int_{t-h_{i}}^{t} x^{\prime}(s) B_{i}^{\prime} P R_{1 i}^{-1} P B_{i} x(s) d s \\
& +\frac{1}{2} \sum_{i=1}^{m} \tau_{i}^{2} x^{\prime}(t) C_{i}^{\prime}\left(R_{2 i}+P R_{2 i}^{-1} P\right) C_{i} x(t)-\frac{1}{2} \sum_{i=1}^{m} \tau_{i} \int_{t-\tau_{i}}^{t} x^{\prime}(s) C_{i}^{\prime}\left(R_{2 i}+P R_{2 i}^{-1} P\right) C_{i} x(s) d s .
\end{aligned}
$$

From (2.6), (2.7) for the functional $V=V_{1}+V_{2}$ we have 


$$
\begin{aligned}
& L V\left(x_{t}\right) \leq x^{\prime}(t) Q x(t), \\
& Q=A^{\prime} P+P A+\sum_{i=1}^{k} h_{i}\left(R_{1 i}+B_{i}^{\prime} P R_{1 i}^{-1} P B_{i}\right)+\frac{1}{2} \sum_{i=1}^{m} \tau_{i}^{2} C_{i}^{\prime}\left(R_{2 i}+P R_{2 i}^{-1} P\right) C_{i} .
\end{aligned}
$$

If the matrix $Q$ is negative definite then the constructed functional $V\left(x_{t}\right)$ satisfies all conditions of Theorem 1.1 and therefore the zero solution of the Eq. (1.1) is asymptotically mean square stable.

Using the matrices $\Phi, \Psi$ and $R$, defined in (2.2), one can represent the matrix $Q$ in the form

$$
\begin{aligned}
Q & =A^{\prime} P+P A+\sum_{i=1}^{k} h_{i} R_{1 i}+\frac{1}{2} \sum_{i=1}^{m} \tau_{i}^{2} C_{i}^{\prime} R_{2 i} C_{i}+\sum_{i=1}^{k} h_{i} B_{i}^{\prime} P R_{1 i}^{-1} P B_{i}+\frac{1}{2} \sum_{i=1}^{m} \tau_{i}^{2} C_{i}^{\prime} P R_{2 i}^{-1} P C_{i} \\
& =\Phi-\Psi R^{-1} \Psi^{\prime} .
\end{aligned}
$$

Via Schur complement the matrix $Q$ is negative definite if and only if the LMI (2.2) holds. The proof is completed.

Remark 2.1 Using Lemma 1.1 with another representation for $a$ and $b$ it is possible to get another $L M I$ in Theorem 2.1 . For example, using $a=P x(t), b=B_{i} x(s)$ for $I_{1 i}$ and $a=x(s), b=C_{i}^{\prime} P C_{i} x(0)$ for $I_{2 i}$, we have

$$
\begin{aligned}
& I_{1 i} \leq h_{i} x^{\prime}(t) P R_{1 i} P x(t)+\int_{t-h_{i}}^{t} x^{\prime}(s) B_{i}^{\prime} R_{1 i}^{-1} B_{i} x(s) d s, \\
& I_{2 i} \leq \frac{\tau_{i}}{2} \int_{t-\tau_{i}}^{t} x^{\prime}(s)\left(R_{2 i}+C_{i}^{\prime} P C_{i} R_{2 i}^{-1} C_{i}^{\prime} P C_{i}\right) x(s) d s .
\end{aligned}
$$

Choosing the additional functional $V_{2}$ in the form

$$
\begin{aligned}
V_{2}\left(x_{t}\right) & =\sum_{i=1}^{k} \int_{t-h_{i}}^{t}\left(s-t+h_{i}\right) x^{\prime}(s) B_{i}^{\prime} R_{1 i}^{-1} B_{i} x(s) d s \\
& +\frac{1}{2} \sum_{i=1}^{m} \tau_{i} \int_{t-\tau_{i}}^{t}\left(s-t+\tau_{i}\right) x^{\prime}(s)\left(R_{2 i}+C_{i}^{\prime} P C_{i} R_{2 i}^{-1} C_{i}^{\prime} P C_{i}\right) x(s) d s
\end{aligned}
$$

instead of (2.8) and (2.2) we obtain the matrix $Q$ and the $L M I$ in another form

$$
\begin{aligned}
Q=A^{\prime} P+P A+\sum_{i=1}^{k} h_{i} P R_{1 i} P+\frac{1}{2} \sum_{i=1}^{m} \tau_{i}^{2} R_{2 i}+\sum_{i=1}^{k} h_{i} B_{i}^{\prime} R_{1 i}^{-1} B_{i}+\frac{1}{2} \sum_{i=1}^{m} \tau_{i}^{2} C_{i}^{\prime} P C_{i} R_{2 i}^{-1} C_{i}^{\prime} P C_{i} \\
=\Phi-\Psi R^{-1} \Psi^{\prime}, \\
{\left[\begin{array}{cc}
\Phi & \Psi \\
\Psi^{\prime} & R
\end{array}\right]<0, \quad \Phi=A^{\prime} P+P A+\sum_{i=1}^{k} h_{i} P R_{1 i} P+\frac{1}{2} \sum_{i=1}^{m} \tau_{i}^{2} R_{2 i} \in \mathbf{R}^{n \times n}, } \\
\Psi=\left[\sqrt{h_{1}} B_{1}^{\prime} \cdots \sqrt{h_{k}} B_{k}^{\prime} \frac{\tau_{1}}{\sqrt{2}} C_{1}^{\prime} P C_{1} \cdots \frac{\tau_{m}}{\sqrt{2}} C_{m}^{\prime} P C_{m}\right] \in \mathbf{R}^{n \times(k+m) n}, \\
R=\operatorname{diag}\left\{-R_{11}, \cdots,-R_{1 k},-R_{21}, \cdots,-R_{2 m}\right\} \in \mathbf{R}^{(k+m) n \times(k+m) n} .
\end{aligned}
$$

Using other different representations for $a$ and $b$, one can get other different forms of the LMI in Theorem 2.1 .

Remark 2.2 In the scalar case both LMIs (2.2) and (2.9) hold if and only if $A+\sum_{i=1}^{k} h_{i}\left|B_{i}\right|+\frac{1}{2} \sum_{i=1}^{m} \tau_{i}^{2} C_{i}^{2}<0$.

\section{Numerical examples}

Here we consider two cases of the stochastic differential equation

$$
d x(t)=\left(A x(t)+\int_{t-h(x(t))}^{t} B x(s) d s\right) d t+\int_{t-\tau}^{t} C x(s) d s d w(t) .
$$

\subsection{System of two equations}

Suppose that in the Eq. (3.1) $0 \leq h(x) \leq h, \tau$ is a constant and

$$
A=\left[\begin{array}{cc}
-3 & 1 \\
1 & -3
\end{array}\right], \quad B=\left[\begin{array}{ll}
0 & 1 \\
1 & 0
\end{array}\right], \quad C=\left[\begin{array}{ll}
1 & 0 \\
0 & 1
\end{array}\right]
$$


The $L M I$ (2.2) takes the form

$$
\left[\begin{array}{ccc}
A^{\prime} P+P A+h R_{1}+\frac{1}{2} \tau^{2} C^{\prime} R_{2} C & \sqrt{h} B^{\prime} P & \frac{\tau}{\sqrt{2}} C^{\prime} P \\
\sqrt{h} P B & -R_{1} & 0 \\
\frac{\tau}{\sqrt{2}} P C & 0 & -R_{2}
\end{array}\right]<0 .
$$

Via MATLAB it was shown that for the matrices (3.2) there exist positive definite matrices $P, R_{1}, R_{2} \in \mathbf{R}^{2 \times 2}$ such that the LMI (3.3) holds, for instance, for $h=1, \tau=0.1$ or for $h=\tau=0.73$ or for $h=0.49, \tau=1$. So, for these values of the delays the zero solution of the Eq. (3.1), (3.2) is asymptotically mean square stable.

\section{2. $A$ scalar equation}

Consider the Eq. (3.1) in the scalar case. Via Remark 2.2, the inequality $\lambda=A+h|B|+\frac{1}{2} \tau^{2} C^{2}<0$ is a sufficient condition for asymptotic mean square stability of the zero solution of the Eq. (3.1).

In Figure 1, 30 trajectories of a solution of the Eq. (3.1) are shown for the following values of the parameters $A=-0.55$, $B=0.4, C=-5, \tau=0.1, h(x)=\frac{1}{1+x^{2}}$, and the initial function $\phi(s)=2 \cos (s), s \in[-1,0]$. In this case, the stability condition $\lambda=-0.025<0$ holds, therefore, the zero solution of the Eq. (3.1) is asymptotically mean square stable and all trajectories converge to zero.

Note that the delay $h(x)=\frac{1}{1+x^{2}}$ satisfies the conditions $h(x) \leq 1$ and $\lim _{x \rightarrow 0} h(x)=1$. In Figure 2,30 trajectories of a solution of the Eq. (3.1) are shown for the same values of the parameters $A, B, C, \tau$ and for the delay $h(x)=\frac{x^{2}}{1+x^{2}}$ that satisfies the conditions $h(x) \leq 1$ and $\lim _{x \rightarrow 0} h(x)=0$.

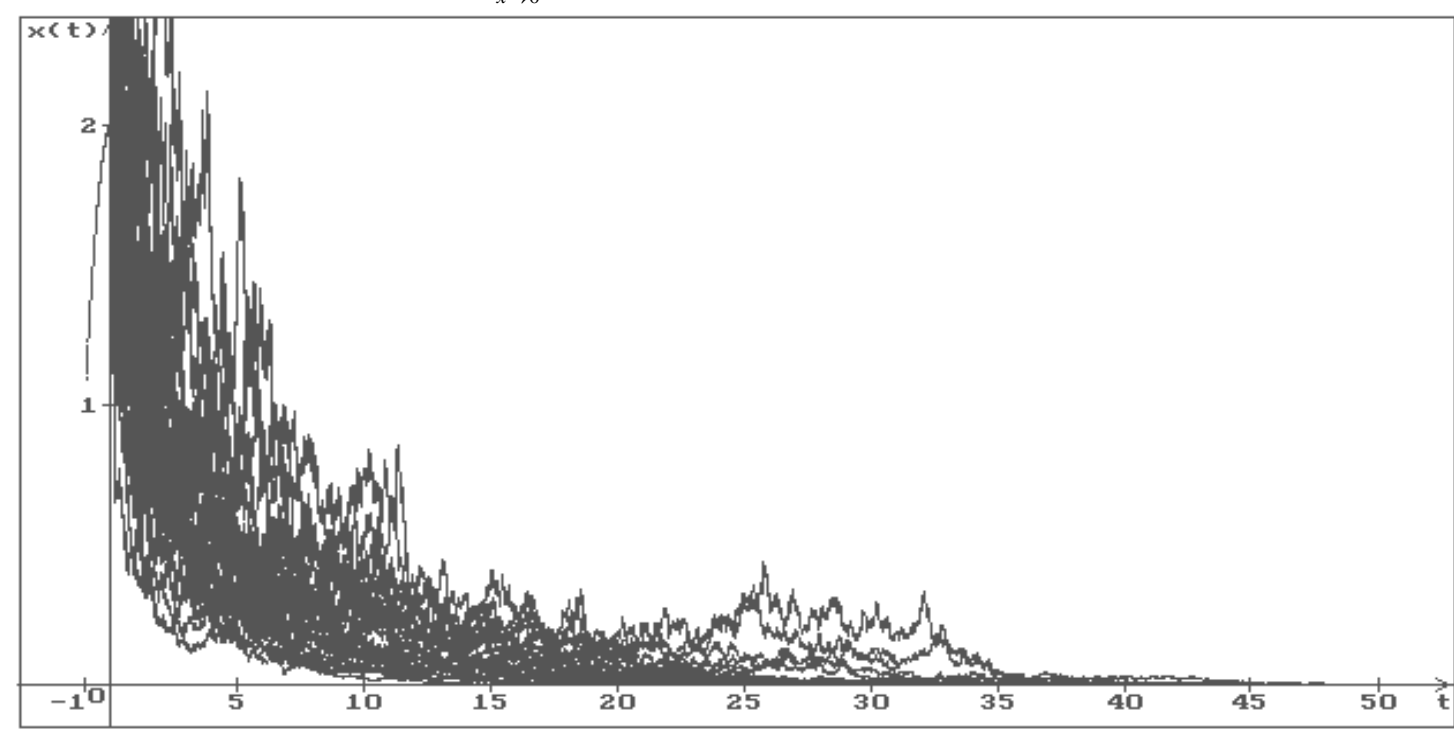

Figure 1. 30 trajectories of the equation (3.1) solution: $A=-0.55, \quad B=0.4, \quad C=-5, \quad \tau=0.1, \quad h(x)=\frac{1}{1+x^{2}}$ 


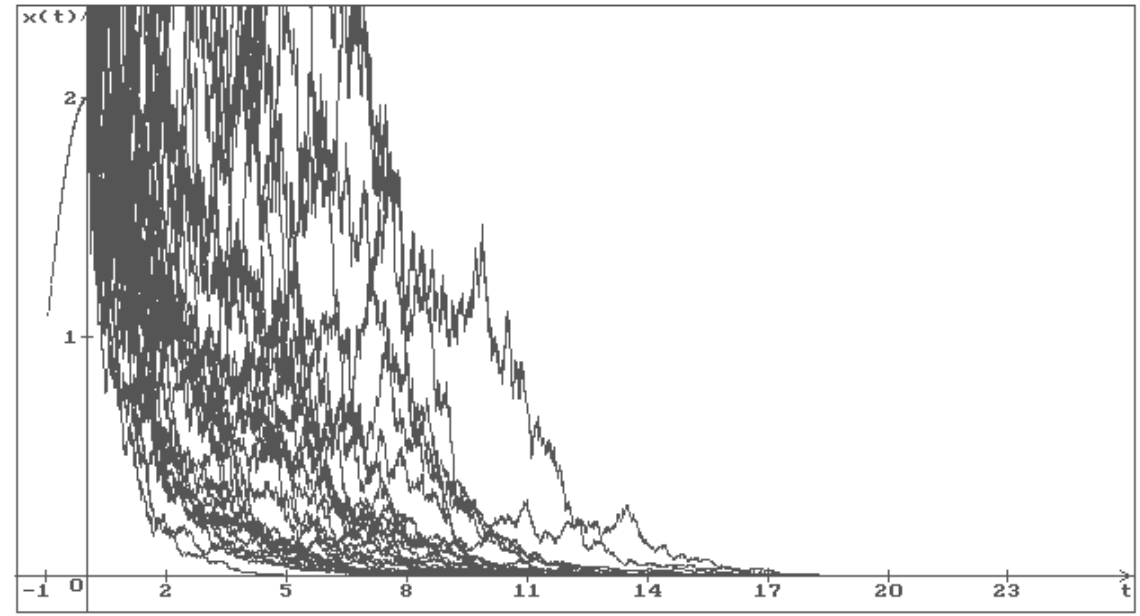

Figure 2. 30 trajectories of the equation (3.1) solution: $A=-0.55, \quad B=0.4, \quad C=-5, \quad \tau=0.1, \quad h(x)=\frac{x^{2}}{1+x^{2}}$

As in the previous case the stability condition $\lambda=-0.025<0$ holds and all trajectories converge to zero. However, one can see that the trajectories of a solution converge to zero essentially quicker than in Figure 1. This is explained by the fact that in the first case, when $x(t)$ tends to zero the delay $h(x(t))$ increases and tends to one, but in the second case, when the $x(t)$ tends to zero, the delay $h(x(t))$ decreases to zero too. Inspite of the fact that in the both cases the condition $h(x) \leq 1$ holds it means that the obtained sufficient stability condition takes into account only the maximum value of the delay but does not take into account the particularity of its behavior.

Consider also Figure 3, where 30 trajectories of a solution of the Eq. (3.1) are shown for the same values of all parameters as in Figure 2 except of $A=-0.5$. In this case $\lambda=0.025>0$, i.e., the stability condition $\lambda<0$ does not hold. Despite of this all trajectories of a solution of the Eq. (3.1) converge to zero too although not as fast as in Figure 2. This fact emphasizes that the obtained stability condition is a sufficient one only and in principle can be improved.

So, we obtain the following

Unsolved problem. To get conditions of asymptotic mean square stability for the zero solution of the Eq. (1.1) that take into account the form of dependence of delays on the system state.

Remark 3.1 Note that by numerical simulation of the Eq. (3.1) solution for numerical simulation of the Wiener process trajectories the algorithm described in [31] was used here.

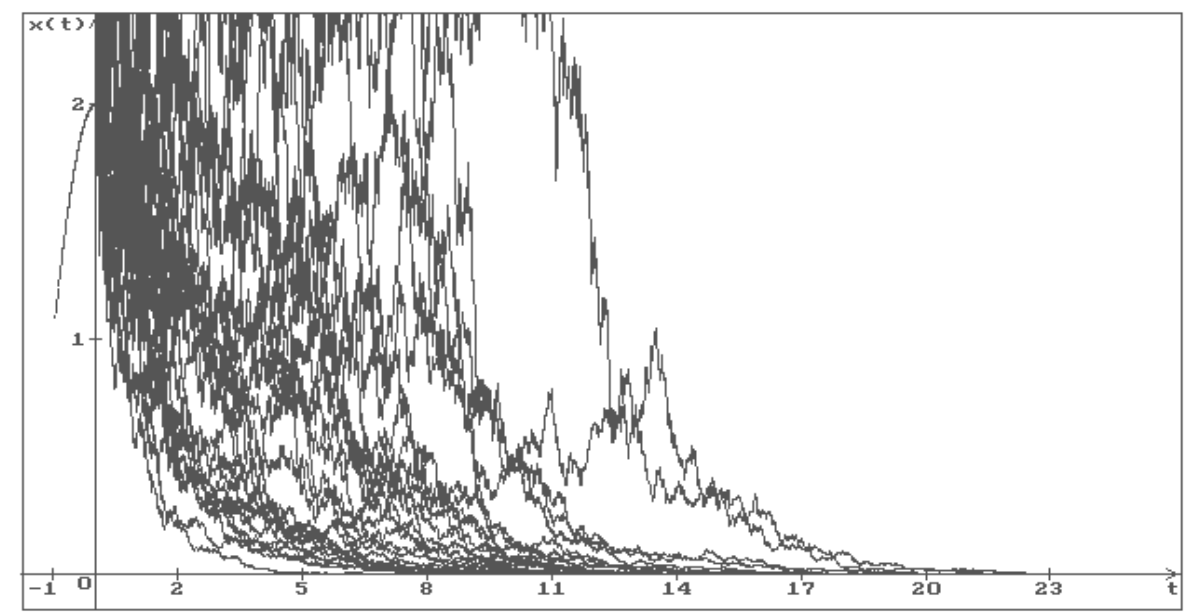

Figure 3. 30 trajectories of the equation (3.1) solution: $A=-0.5, \quad B=0.4, \quad C=-5, \quad \tau=0.1, \quad h(x)=\frac{x^{2}}{1+x^{2}}$ 


\section{Conclusions}

New conditions of asymptotic mean square stability are obtained for a linear stochastic differential equation with distributed and state-dependent delays. Numerical simulations illustrate the obtained results. For future investigation an unsolved problem related to the improvement of the obtained stability conditions is also proposed.

\section{References}

[1] Adimy, M., Crauste, F., Hbid, M. L., Qesmi, R. (2010). Stability and Hopf bifurcation for a cell population model with state-dependent delay. SIAM J. Appl. Math., 70(5), 1611-1633.

[2] Akhtari, B. (2019). Numerical solution of stochastic state-dependent delay differential equations: convergence and stability. Advances in Difference Equations, 2019: 396, 34 pages, https://doi.org/10.1186/s13662-019-2323-x.

[3] Arthi, G., Park, J. H., Jung, H. Y. (2014). Existence and controllability results for second-order impulsive stochastic evolution systems with state-dependent delay. Appl. Math. Comput., 248, 328-341.

[4] Boyd, S., El Ghaoui, L., Feron, E., Balakrishnan, V. (1994). Linear Matrix Inequalities in System and Control Theory. In SIAM Studies in Applied Mathematics, Philadelphia, Pennsylvania: SIAM, 15.

[5] Calleja, B. C., Humphries, A. R., Krauskopf, B. (2017). Resonance phenomena in a scalar delay differential equation with two state-dependent delays. SIAM J. Appl. Dyn. Syst., 16, 1474-1513.

[6] Diblik J. (2020). Long-time behavior of positive solutions of a differential equation with state-dependent delay. Discrete and Continuous Dynamical Systems Series S, 13(1), 31-46.

[7] Diekmann, O., Korvasová, K. (2016). Linearization of solution operators for state-dependent delay equations: a simple example. Discrete and Continuous Dynamical Systems Series A, 36, 137-149.

[8] Domoshnitsky, A., Drakhlin, M., Litsyn, E. (2002). On equations with delay depending on solution. Nonlinear Analysis, 49, 689-701.

[9] Domoshnitsky A., Drakhlin, M., Litsyn, E. (2006). Nonoscillation and positivity of solutions to first order state-dependent differential equations with impulses in variable moments. Journal of Differential Equations, 228, $39-48$.

[10] Fridman, E., Shaikhet, L. (2017). Stabilization by using artificial delays: an LMI approach. Automatica, 81, 429-437.

[11] Getto, Ph, Gyllenberg M., Nakata, Yu, Scarabel F. (2019). Stability analysis of a state-dependent delay differential equation for cell maturation: analytical and numerical methods. Journal of Mathematical Biology, 79(1), 281-328.

[12] Getto Ph, Waurick, M. (2016). A differential equation with state-dependent delay from cell population biology. Journal of Differential Equations, 260(7), 6176-6200.

[13] Gikhman I.I., Skorokhod, A.V. (1972). Stochastic differential equations. Berlin: Springer-Verlag.

[14] Gyori, I., Hartung, F. (2007). Exponential stability of a state-dependent delay system. Discrete and Continuous Dynamical Systems Series A, 18, 773-791.

[15] Hartung, F., Krisztin, T., Walther, H.-O., Wu, J. (2006). Functional differential equations with state-dependent delays: theory and applications, in Handbook of Differential Equations: Ordinary Differential Equations (eds. A. Canada, P. Drabek and A. Fonda), Elsevier/North Holland, 3, 435-545.

[16] Haynsworth, E. V. (1968). On the Schur Complement, in: Basel Mathematical Notes, in: BMN. 20, 17 pages.

[17] Humphries, A. R., Bernucci, D. A., Calleja, R., Homayounfar, N., Snarski, M. (2016). Periodic solutions of a singularly perturbed delay differential equation with two state-dependent delays. Journal of Dynamics and Differential Equations, 28, 1215-1263.

[18] Humphries, A. R., De Masi, O., Magpantay, F. M. G., \& Upham, F. (2012). Dynamics of a delay differential equation with multiple state-dependent delays. Discrete and Continuous Dynamical Systems Series A, 32, 2701-2727.

[19] $\mathrm{Hu}, \mathrm{Q} ., \mathrm{Wu}$, J. (2010). Global Hopf bifurcation for differential equations with state-dependent delay. Journal of Differential Equations, 248(12), 2801-2840.

[20] Insperger, T., Stepan, G., Turi, J. (2007). State-dependent delay in regenerative turning processes, Nonlinear Dynamics, 47, 275-283. 
[21] Kazmerchuk, Y. I., Wu, J. H. (2004). Stochastic state-dependent delay differential equations with applications in finance. Functional Differential Equations, 11(1-2), 77-86.

[22] Kozyreff, G., Erneux, T. (2013). Singular Hopf bifurcation in a differential equation with large state-dependent delay. Proc. Roy. Soc. A, 470, 20130596, 16 pages.

[23] Krisztin, T., Arino, O. (2001). The two-dimensional attractor of a differential equation with state-dependent delay. Journal of Dynamics and Differential Equations, 13, 453-522.

[24] Magpantay, F. M. G., Humphries, A. R. (2020). Generalised Lyapunov-Razumikhin techniques for scalar state-dependent delay differential equations. Discrete and Continuous Dynamical Systems Series S, 13(1), 85-104. doi:10.3934/dcdss.2020005

[25] Magpantay, F. M. G., Kosovalic, N., Wu, J. (2014). An age-structured population model with state-dependent delay: Derivation and numerical integration. SIAM J. Num. Anal., 5(2014), 735-756.

[26] Mallet-Paret, J., Nussbaum, R. D. (1992). Boundary layer phenomena for differential-delay equations with state-dependent time lags, I., Arch. Ration. Mech. Anal., 120(1992), 99-146.

[27] Mallet-Paret, J., Nussbaum, R. D. (1996). Boundary layer phenomena for differential-delay equations with state-dependent time lags, II., J. Reine Angew. Math., 477(1996), 129-197.

[28] Mallet-Paret, J., Nussbaum, R. D. (2003). Boundary layer phenomena for differential-delay equations with state-dependent time lags, III, Discrete and Continuous Dynamical Systems Series A, 189(2003), 640-692.

[29] Mallet-Paret, J., Nussbaum, R. D. (2011). Superstability and rigorous asymptotics in singularly perturbed state-dependent delay-differential equations. Journal of Differential Equations, 250(2011), 4037-4084.

[30] Parthasarathy, C., Mallika Arjunan, M. (2013). Controllability results for first order impulsive stochastic functional differential systems with state-dependent delay. J. Math. Comput. Sci., 3(1), 15-40.

[31] Shaikhet, L. (2013). Lyapunov Functionals and Stability of Stochastic Functional Differential Equations, Springer Science $1 \&$ Business Media.

[32] Stumpf, E. (2016). Local stability analysis of differential equations with state-dependent delay, Discrete and Continuous Dynamical Systems Series A, 36, 3445-3461.

[33] Walther, H.-O. (2009). Algebraic-delay differential systems, state-dependent delay, and temporal order of reactions. Journal of Dynamics and Differential Equations, 21, 195-232.

[34] Walther, H.-O. (2003). The solution manifold and $C^{1}$-smoothness for differential equations with state-dependent delay. Journal of Differential Equations, 195(1), 46-65.

[35] Xu, S., Lam, J. (2008). A survey of linear matrix inequality techniques in stability analysis of delay systems. International Journal of Systems Science, 39(12), 1095-1113.

[36] Zuomao, Y., Lu, F. (2016). Existence and controllability of fractional stochastic neutral functional integro-differential systems with state-dependent delay in Frechet spaces. J. Nonlinear Sci. Appl., 9, 603-616.

[37] Zuomao, Y., Zhang, H. (2013). Existence of solutions to impulsive fractional partial neutral stochastic integro-differential inclusions with state-dependent delay. Electron. Journal of Differential Equations, 2013(81), $1-21$. 